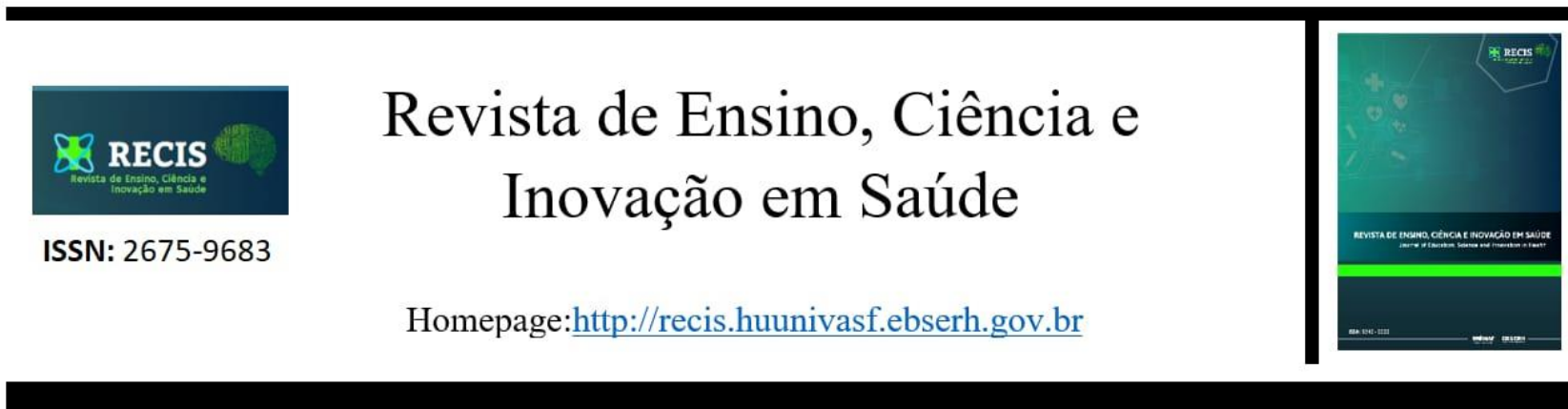

\title{
A realidade virtual na reabilitação do equilíbrio e da marcha em lesões neurológicas: revisão bibliográfica
}

\section{Virtual reality in the rehabilitation of balance and gait in neurological injuries: bibliographic review}

\section{Carolina Cunha Carvalho'; Deysi Micaelli Rodrigues Cantarelli ${ }^{1}$; Pedro Guilherme Campos Lima $^{1}$; Allana Núbia Santos Araújo후; Simone Monteiro de Lima Alves²; Gisele Cunha de Carvalho $^{3}$; Maria Déborah Monteiro de Albuquerque ${ }^{4}$}

${ }^{1}$ Graduandos do curso de Fisioterapia na Faculdade UniBRAS - Juazeiro/BA, ${ }^{2}$ Graduanda do curso de Enfermagem na Faculdade UniBRAS - Juazeiro/BA, ${ }^{3}$ Graduanda do curso de Enfermagem na Faculdade UniFTC - Juazeiro/BA, ${ }^{4}$ Fisioterapeuta e Mestre em Ciências da Saúde e Biológicas da UNIVASF

Autor correspondente: carolina_cunhacarvalho@hotmail.com

Artigo recebido em 19/04/2021e aceito em 13/10/2021

\begin{abstract}
RESUMO
A Realidade Virtual (RV) é uma tecnologia, que proporciona ao usuário uma interação com um sistema operacional, em tempo real, levando a sensações próximas da realidade, com sentimentos de presença. Sendo uma ferramenta cada vez mais utilizada no tratamento fisioterapêutico, surgindo para auxiliar na reabilitação de diversas ocasiões e objetivos, como a reabilitação de indivíduos com lesões neurológicas. O objetivo é realizar um levantamento bibliográfico sobre a eficácia prática da aplicabilidade da RV associada a reabilitação fisioterapêutica no equilíbrio e marcha de indivíduos adultos com lesões neurológicas. Trata-se de uma revisão da literatura, descritiva. A coleta foi realizada em outubro e novembro de 2019, nas bases de dados Scientific Electronic Library Online (SciELO), PubMed, Physiotherapy Evidence Database (PEDro) utilizando referências de 2014 a 2019. Os estudos mostraram diferenças entre os grupos que realizaram a reabilitação associada a RV e os que não utilizaram a RV, pois a RV fornece ambientes que são difíceis de serem executados, fazendo com o que o indivíduo viva novas experiências, estimulando sua neuroplasticidade e o aprendizado motor, por isso se torna mais eficaz do que apenas a reabilitação convencional. De acordo com a literatura, conclui-se que os estudos relatam efeitos positivos no treinamento fisioterapêutico de equilíbrio e marcha associado a RV, além de trazer outros benefícios para os indivíduos, comparado com o tratamento fisioterapêutico convencional.

Palavras-chave: Realidade Virtual; Marcha; Equilíbrio Postural; Doenças do Sistema Nervoso; Modalidades de Fisioterapia.
\end{abstract}

\begin{abstract}
Virtual Reality (VR) is a technology that provides the user with an interaction with an operating system, in real time, leading to sensations close to reality, with feelings of presence. Being an increasingly used tool in physiotherapeutic treatment, arising to assist in the rehabilitation of several occasions and objectives, such as the rehabilitation of individuals with neurological lesions. The objective is to conduct a bibliographic survey on the practical efficacy of VR applicability associated with physiotherapeutic rehabilitation in balance and gait of adult individuals with neurological lesions. This is a review of the literature, descriptive. The collection was carried out in October and November 2019, in the Scientific Electronic Library Online (SciELO), PubMed, Physiotherapy Evidence Database (PEDro) databases using references from 2014 to 2019. The studies showed differences between the groups that underwent rehabilitation associated with VR and those who did not use VR, because VR provides environments that are difficult to perform, causing the individual to
\end{abstract}

Carvalho, C. C. et al. / Revista de Ensino, Ciência e Inovação em Saúde v. 2 n. 3 (2021) p. 26 - 33 
live new experiences, stimulating his/her neuroplasticity and motor learning, so it becomes more effective than just conventional rehabilitation. According to the literature, it is concluded that the studies report positive effects on physiotherapeutic training of balance and gait associated with VR, besides bringing other benefits to individuals, compared with conventional physiotherapeutic treatment.

Keywords: Virtual Reality; Gait; Postural Balance; Nervous System Diseases; Physical Therapy Modalities.

\section{INTRODUÇÃO}

A Realidade Virtual (RV) é definida por simulações interativas criadas por hardware e software para oferecer aos usuários oportunidades de se envolver em ambientes que parecem com objetos e eventos do mundo real. ${ }^{1,2}$

A RV é uma tecnologia, que proporciona ao usuário uma interação com um sistema operacional, em tempo real, levando a sensações próximas da realidade, com sentimentos de presença. ${ }^{1,2}$

Sendo uma ferramenta cada vez mais utilizada, na área da saúde, tem sido aplicada no tratamento fisioterapêutico, surgindo como auxílio na reabilitação de pacientes com doença de Parkinson, esclerose múltipla, pós-Acidente Vascular Cerebral (AVC) agudo e crônico, lesão cerebral traumática e paralisia cerebral. ${ }^{1}$

$\mathrm{Na}$ reabilitação de indivíduos com lesões neurológicas, tem o objetivo de facilitar a aprendizagem motora e promover a neuroplasticidade, fazendo com que esses indivíduos sejam sujeitos a novas experiências ou atividades antigas, já aprendidas anteriormente onde não realizam certos movimentos ou que precisam ser aprimoradas. ${ }^{1,2}$

Os indivíduos que possuem uma lesão neurológica, apresentam deficiências sensoriais, motoras, cognitivas e visuais, levando a comprometimentos que podem ser representadas por perda na força e coordenação muscular, onde sendo acometido os membros inferiores podem ter uma perda/déficit de equilíbrio e limitação na marcha que são itens importantes para uma independência funcional, sendo associado a riscos de queda, diminuição das atividades diárias e consequentemente reduzindo a sua qualidade de vida, principalmente em indivíduos que tenham uma idade mais avançada pois o envelhecimento já leva a perdas de nível funcional e cognitivos. ${ }^{1-4}$

As intervenções utilizadas nesse grupo de indivíduos devem ser adaptadas individualmente de acordo com sua deficiência, ou seja, melhorar suas habilidades sensoriais, motoras e cognitivas, que inclui aprimorar o equilíbrio e a marcha. ${ }^{1.5}$

A marcha é o movimento do corpo que tem como objetivo se deslocar de um ponto para o outro, o comprometimento desse movimento nos indivíduos com lesões neurológicas é o principal motivo para sua falta de independência. Para Gupta \& Taly (2019), os fatores que contribuem para a anormalidade da marcha incluem déficits sensoriais, fraqueza muscular, espasticidade e cognição prejudicada. Esse déficit no caminhar afeta sua funcionalidade, levando a uma baixa velocidade da marcha, resistência e equilíbrio prejudicados, aumenta o risco de quedas e fraturas, comprometendo a qualidade de vida. Por isso, é importante a análise da marcha para realizar o planejamento de tratamentos e intervenções precisos, alcançando os objetivos do tratamento. ${ }^{6-8}$

Para alcançar esses objetivos é necessário fornecer treinamento repetitivo e personalizado de alta intensidade, retransmitir informações sobre o desempenho dos pacientes por meio de feedback multimodal e melhorar a motivação. ${ }^{1,5}$

As vantagens da RV comparando com a reabilitação convencional nesses indivíduos, é o aumento da motivação facilitando o aprimoramento do aprendizado motor, da variação das tarefas, fornecendo ambientes que simulam uma atividade de vida diária (AVD) com feedback multissensorial (visual e auditivo, principalmente) em tempo real, e da progressão do tratamento, pois pode reorganizar a estrutura neural estimulando a recuperação ou o ganho de habilidades motoras, após uma lesão neurológica. ${ }^{1,2}$

De acordo com a literatura já existe uma crescente evidência sobre os benefícios da RV para aperfeiçoar os ganhos adquiridos no equilíbrio e na marcha de indivíduos com lesões neurológicas, além de demostrar ser seguro e econômico, porém ainda não se sabem a dosagem, tarefas adequadas e eficácia a longo prazo, dificultando as intervenções padronizadas no uso da RV na reabilitação.

Este estudo analisa o que há de mais atual na literatura sobre o uso da RV contribuindo na reabilitação fisioterapêutica do equilíbrio e da marcha de indivíduos adultos com lesões neurológicas. $\mathrm{O}$ objetivo é realizar um levantamento bibliográfico sobre o efeito prático da aplicabilidade da RV de indivíduos adultos com lesões neurológicas. 
Revista de Ensino, Ciência e Inovação em Saúde v.2, n.3 (2021) 26 - 33

ISSN: 2675-9683/DOI: 10.51909/recis.v2i3.142

\section{MATERIAL E MÉTODOS}

\section{Fontes de dados e pesquisas}

Foi realizada uma pesquisa bibliográfica em outubro e novembro de 2019, nas bases de dados Scientific Electronic Library Online (SciELO), PubMed, Physiotherapy Evidence Database (PEDro) utilizando referências de 2014 a 2019. Os termos de pesquisa incluíram palavraschave na língua portuguesa como "Realidade Virtual" and/or "Marcha" and "Equilíbrio Postural" and "Doenças do Sistema Nervoso" and "Modalidades de Fisioterapia". Na língua inglesa com os termos: "Virtual Reality" and "Gait" and "Postural Balance" and "Nervous System Diseases" and "Physical Therapy Modalities".

\section{Seleção do estudo}

Foram incluídos Ensaios Clínicos/ Controlados Randomizados (ECRs), voltados para a população adulta ou idosa, acima de 18 anos, com sequelas de doenças neurológicas como a doença de Parkinson, esclerose múltipla, pós-AVC agudo e crônico.
O presente trabalho se deteve em pesquisar apenas estudos com intervenções fisioterápicas que compararam o efeito do treinamento de marcha ou equilíbrio sem RV com o efeito de treinamento de marcha ou equilíbrio com RV, ou que abordavam o efeito da RV voltada para o tratamento de indivíduos com lesões neurológicas.

Também para inclusão todos os artigos deveriam estar completos e escritos na língua portuguesa ou inglesa e como o objetivo desta revisão de literatura é determinar o que há de mais atual da implementação da RV na reabilitação.

Foram excluídos consequentemente artigos voltados para a população pediátrica, além disso, estudos como revisões sistemáticas e metaanálises e artigos duplicados.

\section{Identificação dos artigos encontrados}

No total, 25 artigos foram encontrados nas bases de dados escolhidas, onde 2 eram artigos duplicados, sendo removidos e 14 também foram removidos pois não cumpriam com os critérios de inclusão do estudo. Com isso, 9 artigos foram incluídos na revisão. Como mostra no Fluxograma 1.

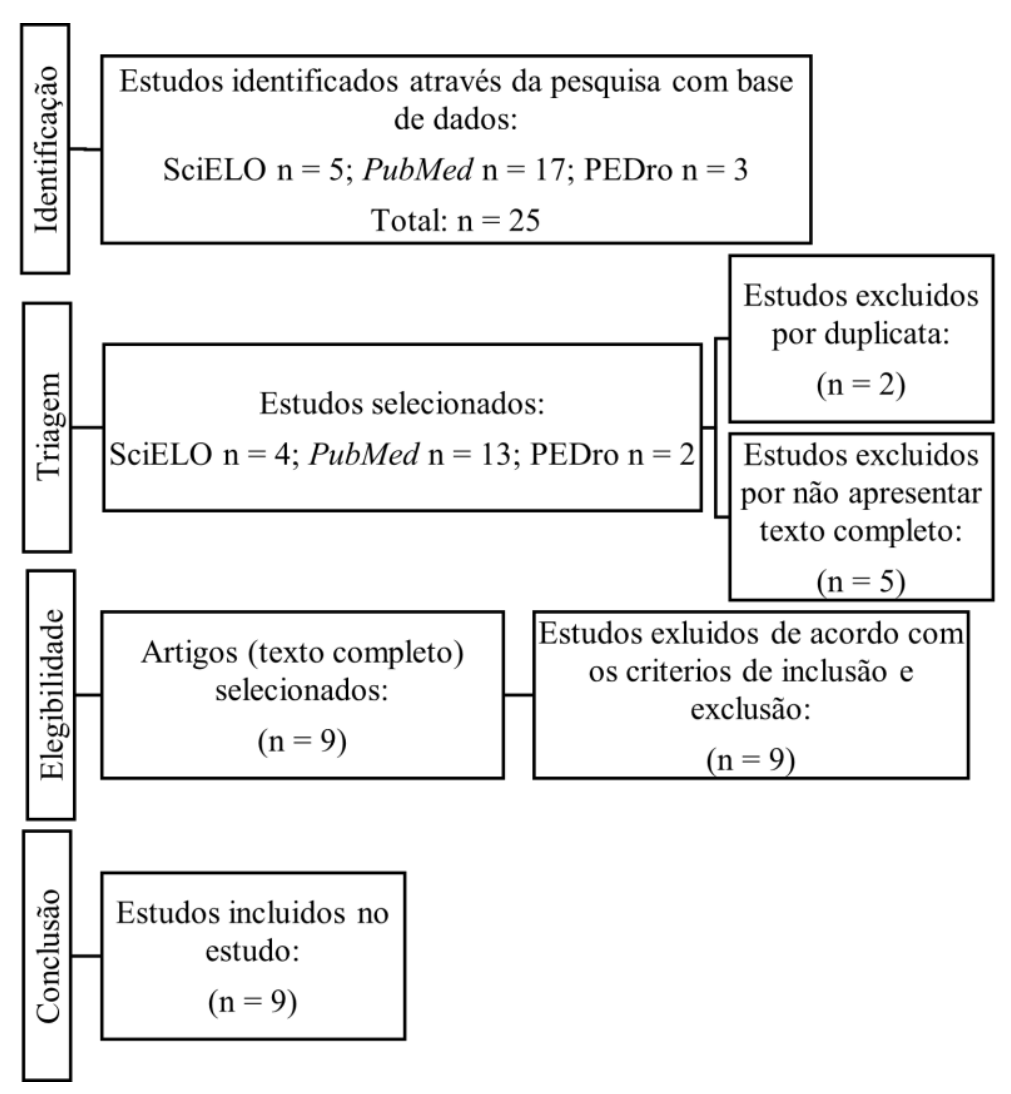

Fluxograma 1 - Seleção e inclusão dos artigos no estudo

Carvalho, C. C. et al. / Revista de Ensino, Ciência e Inovação em Saúde v. 2 n. 3 (2021) p. 26 - 33 
Revista de Ensino, Ciência e Inovação em Saúde v.2, n.3 (2021) 26 - 33

ISSN: 2675-9683/DOI: $10.51909 /$ recis.v2i3.142

Tabela 1 - Características dos estudos selecionados

\section{$\begin{array}{cccccc}\text { AUTOR } & \text { DESENHO } & \text { OBJETIVO } & \text { POPULAÇÃO } & \text { INTERVENÇÃO } & \text { DESFECHO } \\ \text { (ANO) } & \text { DO ESTUDO } & \text { OBJERT } & \end{array}$}

\begin{tabular}{|c|c|c|c|c|}
\hline Bergmann $^{9}$ & $\begin{array}{c}\text { Ensaio piloto } \\
\text { randomizado, } \\
\text { cego, } \\
\text { controlado, } \\
\text { com dois } \\
\text { braços } \\
\text { paralelos }\end{array}$ & $\begin{array}{c}\text { Avaliar a } \\
\text { aceitabilidad } \\
\text { e do } \\
\text { treinamento } \\
\text { de marcha } \\
\text { assistida por } \\
\text { robô } \\
\text { (RAGT) com } \\
\text { e sem RV }\end{array}$ & $\begin{array}{c}20 \text { pacientes } \\
\text { com AVC } \\
\text { subagudo, sexo } \\
\text { masculino e } \\
\text { feminino e idade } \\
\text { entre } 64 \text { a } 69 \\
\text { anos }\end{array}$ & $\begin{array}{l}\text { Doze sessões de } \\
\text { RAGT aumentado } \\
\text { com RV (grupo de } \\
\text { intervenção) ou } \\
\text { RAGT padrão, sem } \\
\text { RV (grupo de } \\
\text { controle) }\end{array}$ \\
\hline
\end{tabular}

$\mathrm{O}$ treinamento

de marcha assistida

por robô

aumentado por $\mathrm{RV}$

resultou em alta

aceitabilidade e

motivação, além de uma taxa de

abandono reduzida

e um tempo de

treinamento

prolongado em

comparação ao

treinamento

de marcha assistida

por robô padrão, sem RV

\begin{tabular}{|c|c|c|c|c|c|}
\hline Rooij $^{10}$ & $\begin{array}{c}\text { Ensaio clínico } \\
\text { cego, } \\
\text { randomizado e } \\
\text { controlado } \\
\text { com dois } \\
\text { grupos } \\
\text { paralelos }\end{array}$ & $\begin{array}{l}\text { Examinar o } \\
\text { efeito do } \\
\text { treinamento } \\
\text { de marcha } \\
\text { com RV na } \\
\text { participação } \\
\text { de pessoas } \\
\text { que vivem na } \\
\text { comunidade } \\
\text { após o AVC }\end{array}$ & $\begin{array}{l}\text { Pacientes pós- } \\
\text { AVC subagudo, } \\
\text { sexo masculino } \\
\text { e feminino e } \\
\text { idade entre } 18 \text { a } \\
80 \text { anos }\end{array}$ & $\begin{array}{l}\text { Treino de marcha } \\
\text { em realidade virtual } \\
\text { ou grupo de treino } \\
\text { de marcha em } \\
\text { realidade não } \\
\text { virtual, que consiste } \\
\text { em treinamento } \\
\text { convencional em } \\
\text { esteira e exercícios } \\
\text { funcionais de } \\
\text { marcha, durante } 2 \\
\text { sessões de } 30 \\
\text { minutos por } \\
\text { semana, por } 6 \\
\text { semanas (12 } \\
\text { sessões) }\end{array}$ & $\begin{array}{c}\text { O treinamento de } \\
\text { marcha com RV é } \\
\text { um grande } \\
\text { potencial para } \\
\text { reabilitação após o } \\
\text { AVC, sendo uma } \\
\text { adição valiosa à } \\
\text { fisioterapia, } \\
\text { fornecendo uma } \\
\text { terapia intensiva, } \\
\text { variável e } \\
\text { agradável que pode } \\
\text { ser facilmente } \\
\text { ajustada às } \\
\text { habilidades do } \\
\text { paciente }\end{array}$ \\
\hline $\operatorname{In}^{11}$ & $\begin{array}{c}\text { Ensaio clínico } \\
\text { randomizado }\end{array}$ & $\begin{array}{c}\text { Investigar se } \\
\text { a terapia de } \\
\text { reflexão de } \\
\text { RV poderia } \\
\text { melhorar o } \\
\text { equilíbrio } \\
\text { postural e a } \\
\text { habilidade de } \\
\text { marcha de } \\
\text { pacientes } \\
\text { com AVC } \\
\text { crônico }\end{array}$ & $\begin{array}{l}25 \text { pacientes } \\
\text { pós-AVC } \\
\text { crônico }\end{array}$ & $\begin{array}{l}\text { Um grupo utilizou } \\
\text { a terapia de } \\
\text { reflexão de } \mathrm{RV} \text { e o } \\
\text { grupo controle a } \\
\text { terapia } \\
\text { convencional, } \\
\text { durante cinco vezes } \\
\text { por semana, por } 4 \\
\text { semanas }\end{array}$ & $\begin{array}{c}\text { A aplicação da } \\
\text { terapia de reflexão } \\
\text { de RV junto com } \\
\text { um programa de } \\
\text { reabilitação } \\
\text { convencional para } \\
\text { pacientes com } \\
\text { AVC crônico é } \\
\text { ainda mais benéfico } \\
\text { do que o programa } \\
\text { de reabilitação } \\
\text { convencional } \\
\text { sozinho para } \\
\text { melhorar a função } \\
\text { afetada dos } \\
\text { membros inferiores }\end{array}$ \\
\hline
\end{tabular}


Revista de Ensino, Ciência e Inovação em Saúde v.2, n.3 (2021) 26 - 33

ISSN: 2675-9683/DOI: 10.51909/recis.v2i3.142

\begin{tabular}{|c|c|c|c|c|c|}
\hline Kalron $^{12}$ & $\begin{array}{l}\text { Ensaio piloto } \\
\text { randomizado } \\
\text { controlado }\end{array}$ & $\begin{array}{c}\text { Examinar a } \\
\text { eficácia de } \\
\text { um programa } \\
\text { de } \\
\text { treinamento } \\
\text { de equilíbrio } \\
\text { de RV de } 6 \\
\text { semanas }\end{array}$ & $\begin{array}{l}32 \text { pessoas com } \\
\text { Esclerose } \\
\text { Múltipla } \\
\text { remitente- } \\
\text { recorrente }\end{array}$ & $\begin{array}{c}\text { Grupo de } \\
\text { intervenção usando } \\
\text { a RV e o grupo de } \\
\text { controle. Cada } \\
\text { grupo recebeu } \\
\text { sessões de } \\
\text { treinamento de } \\
\text { equilíbrio por } 6 \\
\text { semanas } \\
\text { consecutivas, duas } \\
\text { sessões por semana, } \\
\text { sessões de } 30 \\
\text { minutos }\end{array}$ & $\begin{array}{l}\text { O treinamento de } \\
\text { equilíbrio baseado } \\
\text { no dispositivo é um } \\
\text { método eficaz de } \\
\text { treinamento de } \\
\text { equilíbrio para } \\
\text { pacientes com } \\
\text { Esclerose Múltipla }\end{array}$ \\
\hline $\mathrm{Kim}^{13}$ & $\begin{array}{c}\text { Estudo } \\
\text { comparativo }\end{array}$ & $\begin{array}{l}\text { Determinar } \\
\text { se idosos e } \\
\text { indivíduos } \\
\text { com DP } \\
\text { podem usar a } \\
\text { RV, por um } \\
\text { longo } \\
\text { período de } \\
\text { caminhada } \\
\text { sem efeitos } \\
\text { adversos }\end{array}$ & $\begin{array}{l}33 \text { participantes, } \\
\text { sendo } 11 \text { jovens } \\
\text { saudáveis ( } 27 \text { a } \\
28 \text { anos), } 11 \\
\text { idosos saudáveis } \\
\text { (63 a } 66 \text { anos) e } \\
11 \text { indivíduos } \\
\text { com a doença de } \\
\text { Parkinson (65 a } \\
67 \text { anos) }\end{array}$ & $\begin{array}{l}\text { Os participantes } \\
\text { caminharam por } 20 \\
\text { minutos enquanto } \\
\text { visualizavam uma } \\
\text { cena de cidade } \\
\text { virtual por meio de } \\
\text { um HMD (Oculus } \\
\text { Rift DK2). A } \\
\text { segurança foi } \\
\text { avaliada usando o } \\
\text { mini-BESTest, } \\
\text { medidas de } \\
\text { excursão do centro } \\
\text { de pressão (CoP) e } \\
\text { questionários } \\
\text { abordando sintomas } \\
\text { de doença do } \\
\text { simulador (SSQ) e } \\
\text { medidas de estresse } \\
\text { e excitação. } \\
\end{array}$ & $\begin{array}{l}\text { Adultos mais } \\
\text { velhos e indivíduos } \\
\text { com a doença de } \\
\text { Parkinson foram } \\
\text { capazes de usar a } \\
\text { RV imersiva } \\
\text { durante a } \\
\text { caminhada sem } \\
\text { efeitos adversos, } \\
\text { comprovando a } \\
\text { segurança da RV } \\
\text { imersiva para o } \\
\text { treinamento da } \\
\text { marcha nessas } \\
\text { populações. }\end{array}$ \\
\hline $\mathrm{Liao}^{4}$ & $\begin{array}{l}\text { Ensaio de } \\
\text { controle } \\
\text { randomizado }\end{array}$ & $\begin{array}{l}\text { Avaliar os } \\
\text { efeitos do } \\
\text { treinamento } \\
\text { físico e } \\
\text { cognitivo } \\
\text { baseado em } \\
\text { RV na } \\
\text { função } \\
\text { executiva e } \\
\text { desempenho } \\
\text { de marcha } \\
\text { em dupla } \\
\text { tarefa em } \\
\text { adultos mais } \\
\text { velhos com } \\
\text { deficiência } \\
\text { cognitiva } \\
\text { leve }\end{array}$ & $\begin{array}{l}\text { Trinta e quatro } \\
\text { idosos, acima de } \\
65 \text { anos, com } \\
\text { comprometimen } \\
\text { to cognitivo leve }\end{array}$ & $\begin{array}{c}\text { Treinamento físico } \\
\text { e cognitivo baseado } \\
\text { em RV ou } \\
\text { tradicional por } 36 \\
\text { sessões durante } 12 \\
\text { semanas }\end{array}$ & $\begin{array}{c}\text { O programa levou a } \\
\text { melhorias } \\
\text { significativas no } \\
\text { desempenho da } \\
\text { marcha em dupla } \\
\text { tarefa em idosos, } \\
\text { como na velocidade } \\
(\mathrm{cm} / \mathrm{s}) \text {, que de } 26,9 \\
\text { a } 68,1 \text { foi para } 30,6 \\
\text { a } 82,5 \text { depois da } \\
\text { intervenção, o que } \\
\text { pode ser atribuído a } \\
\text { melhorias na } \\
\text { função executada }\end{array}$ \\
\hline
\end{tabular}


Revista de Ensino, Ciência e Inovação em Saúde v.2, n.3 (2021) 26 - 33

ISSN: 2675-9683/DOI: 10.51909/recis.v2i3.142

\begin{tabular}{|c|c|c|c|c|c|}
\hline $\mathrm{Oh}^{7}$ & $\begin{array}{c}\text { Avaliação } \\
\text { Biomecânica }\end{array}$ & $\begin{array}{l}\text { Verificar se é } \\
\text { possível } \\
\text { iniciar o giro } \\
\text { em uma } \\
\text { esteira linear } \\
\text { com ritmo } \\
\text { próprio } \\
\text { baseado em } \\
\text { RV }\end{array}$ & $\begin{array}{c}\text { Pacientes } \\
\text { adultos, de } 22 \text { a } \\
35 \text { anos, com } \\
\text { lesões cerebrais }\end{array}$ & $\begin{array}{c}\text { Caminhada baseada } \\
\text { em RV combinada } \\
\text { com a esteira } \\
\text { unidirecional para } \\
\text { estimular o usuário } \\
\text { a iniciar a marcha } \\
\text { de giro }\end{array}$ & $\begin{array}{l}\text { Houve aumentos } \\
\text { significativos na } \\
\text { rotação da cabeça, } \\
\text { rotação pélvica, } \\
\text { abdução do quadril } \\
\text { direito, adução do } \\
\text { quadril esquerdo, } \\
\text { progressão do pé, } \\
\text { forças de reação } \\
\text { medial-lateral do } \\
\text { solo, nível de } \\
\text { ativação do } \\
\text { isquiotibial medial } \\
\text { direito e alterações } \\
\text { na largura do passo } \\
\text { durante a RV em } \\
\text { comparação com a } \\
\text { caminhada em } \\
\text { linha reta }\end{array}$ \\
\hline Severiano $^{14}$ & $\begin{array}{c}\text { Estudo } \\
\text { observacional } \\
\text { prospectivo }\end{array}$ & $\begin{array}{l}\text { Verificar a } \\
\text { eficácia dos } \\
\text { exercícios de } \\
\text { equilíbrio } \\
\text { com } \\
\text { realidade } \\
\text { virtual na } \\
\text { doença de } \\
\text { Parkinson }\end{array}$ & $\begin{array}{l}6 \text { pacientes do } \\
\text { sexo feminino e } \\
10 \text { do sexo } \\
\text { masculino com } \\
\text { a doença de } \\
\text { Parkinson, com } \\
\text { idade entre } 18 \text { e } \\
82 \text { anos }\end{array}$ & $\begin{array}{l}\text { Foram submetidos } \\
\text { a testes e escalas de } \\
\text { equilíbrio, antes e } \\
\text { depois da aplicação } \\
\text { da realidade virtual }\end{array}$ & $\begin{array}{l}\text { A reabilitação do } \\
\text { equilíbrio corporal } \\
\text { por meio da RV } \\
\text { mostrou-se } \\
\text { eficiente para } \\
\text { melhorar o } \\
\text { equilíbrio corporal } \\
\text { e a capacidade } \\
\text { funcional, } \\
\text { reduzindo o risco } \\
\text { de quedas, } \\
\text { aumentando a } \\
\text { autoconfiança e } \\
\text { melhorando a } \\
\text { qualidade de vida } \\
\text { desses pacientes }\end{array}$ \\
\hline Yang ${ }^{15}$ & $\begin{array}{l}\text { Ensaio clínico } \\
\text { randomizado }\end{array}$ & $\begin{array}{c}\text { Testar a } \\
\text { eficácia do } \\
\text { treinamento } \\
\text { de equilíbrio } \\
\text { de realidade } \\
\text { virtual em } \\
\text { casa na } \\
\text { melhoria do } \\
\text { equilíbrio, } \\
\text { caminhada e } \\
\text { qualidade de } \\
\text { vida em } \\
\text { pacientes } \\
\text { com doença } \\
\text { de Parkinson } \\
\text { (DP). }\end{array}$ & $\begin{array}{c}23 \text { pacientes } \\
\text { com doença de } \\
\text { Parkinson, com } \\
\text { idade de } 55 \text { a } 85 \\
\text { anos, função } \\
\text { cognitiva } \\
\text { intacta, sem } \\
\text { praticar } \\
\text { exercícios de } \\
\text { equilíbrio ou } \\
\text { marcha nos } \\
\text { últimos } 6 \text { meses } \\
\text { e sem nenhuma } \\
\text { condição médica } \\
\text { não tratada que } \\
\text { possa afetar o } \\
\text { equilíbrio e a } \\
\text { função de } \\
\text { caminhar }\end{array}$ & $\begin{array}{c}\text { Foram realizadas } \\
\text { doze sessões de } \\
\text { treinamento de } 50 \\
\text { minutos durante o } \\
\text { período de } \\
\text { treinamento de } 6 \\
\text { semanas. O grupo } \\
\text { experimental foi } \\
\text { treinado com um } \\
\text { sistema de } \\
\text { treinamento de } \\
\text { equilíbrio de } \\
\text { realidade virtual, e } \\
\text { o grupo controle foi } \\
\text { treinado com } \\
\text { fisioterapia } \\
\text { convencional }\end{array}$ & $\begin{array}{l}\text { Este estudo não } \\
\text { encontrou nenhuma } \\
\text { diferença entre os } \\
\text { efeitos do } \\
\text { treinamento de } \\
\text { equilíbrio da RV } \\
\text { em casa e do } \\
\text { treinamento } \\
\text { convencional de } \\
\text { equilíbrio em casa, } \\
\text { as duas opções de } \\
\text { treinamento foram } \\
\text { eficazes }\end{array}$ \\
\hline
\end{tabular}




\section{DESENVOLVIMENTO}

Os efeitos dos programas de reabilitação associado a RV de acordo com os estudos incluídos

De acordo com a literatura indivíduos com lesões neurológicas têm dificuldades durante as transições da marcha, como início, término e giro da etapa, demostrando um aumento do tremor, hesitação, perda de estabilidade ou até quedas, que costumam causas outras lesões, demostrando a importância de incluir a marcha e o equilíbrio na reabilitação fisioterapêutica desses indivíduos. ${ }^{7}$

Os estudos mostraram diferenças entre os grupos que realizaram a reabilitação associada a $\mathrm{RV}$ e os que não utilizaram, pois, a RV fornece ambientes que são difíceis de serem executados, fazendo com o que o indivíduo viva novas experiências, estimulando sua neuroplasticidade e o aprendizado motor, por isso se torna mais eficaz do que apenas a reabilitação convencional..$^{9,10}$

Segundo a literatura o tratamento com o uso da RV mostrou-se ser seguro, econômico, variável, agradável e eficaz para esse tipo de indivíduo, beneficiando com motivações claras, integrando vários estímulos sensoriais através de sistemas visual, auditivo, tátil e somatossensorial, melhorando o efeito do tratamento. Além de fornecer informações diferentes para o indivíduo: A)Visualização de movimento: os movimentos do paciente são capturados e representados no ambiente virtual, durante uma atividade motora; B)Feedback de desempenho: as informações sobre o objetivo atingido ou uma alteração de movimento necessária são transmitidas através de uma ou várias modalidades sensoriais, de acordo com a conclusão da tarefa; C)Informações de contexto: os recursos são incorporados em um ambiente virtual, onde pode variar de um ambiente técnico muito realista a um irrealista. ${ }^{4,7,9-15}$

A RV foi benéfica pois teve aumentos significativos na rotação da cabeça, rotação pélvica, abdução do quadril direito, adução do quadril esquerdo, progressão do pé, forças de reação medial-lateral do solo, nível de ativação do isquiotibial medial direito e alterações na largura do passo durante a RV em comparação com a caminhada em linha reta. ${ }^{7}$

A RV associada a reabilitação teve benefícios, principalmente quando combinado com outras intervenções, como: melhora na velocidade da marcha durante condições de tarefa única e dupla, na mobilidade funcional com carga cognitiva, no equilíbrio dinâmico, no ganho de força muscular e amplitude de movimento articular e diminuindo a dor e o risco de queda, aumentando a qualidade de vida e a capacidade de realizar as atividades de vida diária. ${ }^{4,7,9-15}$

Para os pesquisadores o uso da RV é viável e eficaz quando implementado de maneira clínica rotineira, isso porque concluiu que a reabilitação baseada em RV traz vantagens sobre a reabilitação convencional por incorporar princípios de aprendizado motor, porém existem muitos desafios relacionados à incorporação da tecnologia da RV como as ferramenta terapêutica de rotina, principalmente devido ao número relativamente grande de graus de liberdade que a tecnologia permite, por exemplo, tipos de ambientes e cenários de feedback. ${ }^{4}$

É importante salientar que a base para o aprendizado motor é o treinamento repetitivo, o que a RV disponibiliza com mais facilidade, beneficiando o aperfeiçoamento de habilidades motoras pelo treinamento motor específico, havendo diferença, nos estudos incluídos, na intensidade imposta no treinamento durante a reabilitação, podendo ser ajustada de acordo com as necessidades e características do indivíduo. As mudanças durante o treinamento, também são importantes pois ajudará a melhorar a capacidade de adaptação a novas situações. ${ }^{9,11,12}$

O feedback, ou seja, a reação do indivíduo ao estimulo durante a utilização da RV pode ser dividida em intrínseco e extrínseco, sabendo-se que o feedback melhora a capacidade de aprendizagem. $\mathrm{O}$ feedback intrínseco refere-se a informações somáticas, incluindo informações táteis, proprioceptivas e cinestésicas, já o feedback extrínseco é fornecido através de uma fonte externa. ${ }^{9,11,12}$

Com isso, acredita-se que a RV melhora todos os aspectos envolvidos na reabilitação do equilíbrio e da marcha de indivíduos com lesões neurológicas, melhorando a motivação, o prazer, a aceitação do tratamento e diminuindo a percepção do esforço.

\section{CONCLUSÃO}

De acordo com a literatura, conclui-se que os estudos relatam efeitos positivos no treinamento fisioterapêutico de equilíbrio e marcha associado a $\mathrm{RV}$, além de trazer outros benefícios para os indivíduos, comparado com o tratamento fisioterapêutico convencional. Sendo indivíduos de difíceis tratamento, são necessários as adaptações e

Carvalho, C. C. et al. / Revista de Ensino, Ciência e Inovação em Saúde v. 2 n. 3 (2021) p. 26 - 33 
Revista de Ensino, Ciência e Inovação em Saúde v.2, n.3 (2021) 26 - 33

ISSN: 2675-9683/DOI: 10.51909/recis.v2i3.142

os detalhes a serem usados devem ser definidos de acordo com os objetivos a serem alcançados.

\section{REFERÊNCIAS BIBLIOGRÁFICAS}

1. Porras DC, Sharon H, Inzelberg R, Ziv-Ner Y, Zeilig G, Plotnik M. Reabilitação avançada de equilíbrio e marcha baseada em realidade virtual na prática clínica. Avanços terapêuticos em doenças crônicas, 10, 2040622319868379. (2019). https://doi.org/10.1177/20406223198 68379

2. Lee HS, Park YJ, Park SW. The Effects of Virtual Reality Training on Function in Chronic Stroke Patients: A Systematic Review and Meta-Analysis. BioMed Research International, 1-12. (2019)

3. Rooij IJMD, Port IGLVD, Meily JMAV, Meijer JWG. Virtual reality gait training versus non-virtual reality gait training for improving participation in subacute stroke survivors: study protocol of the ViRTAS randomized controlled trial. Trials. 20(1), 89-. (2019). https://doi:10.1186/s13063-018-3165-7

4. Liao YY, Chen IH, Lin YJ, Chen Y, Hsu WC. Effects of Virtual Reality-Based Physical and Cognitive Training on Executive Function and Dual-Task Gait Performance in Older Adults With Mild Cognitive Impairment: A Randomized Control Trial. Frontiers in Aging Neuroscience, 11, $162 . \quad$ (2019) https://doi:10.3389/fnagi.2019.00162

5. Santos LFD, Christ O, Mate K, Schmidt H, Krüger J, Dohle C. Visualização do movimento na reabilitação de realidade virtual do membro inferior: uma revisão sistemática. BioMed Eng OnLine 15, 144 (2016). https://doi.org/10.1186/s12938-0160289-4

6. Gupta A, Taly AB. Post-stroke Gait Analysis in Rehabilitation Set-up: Observational or Instrumental!. Neurology India, 67(4), 10411042 (2019). https://doi.org/10.4103/00283886.266276

7. Oh K, Stanley CL, Damiano DL, Kim J, Yoon J, Park HS. Biomechanical Evaluation of Virtual Reality-based Turning on a Self-Paced Linear Treadmill. Gait \& posture vol. 65 157162. (2018). https://doi:10.1016/j.gaitpost.2018.07.175

8. Sattelmayer M, Chevalley O, Steuri R, Hilfiker R. Over-ground walking or robot-assisted gait training in people with .multiple sclerosis: does the effect depend on baseline walking speed and disease related disabilities? A systematic review and meta-regression. $B M C$ neurology, 19(1), 93. https://doi:10.1186/s12883-019-1321-7

9. Bergmann J, Krewer C, Bauer P, Koenig A, Riener R, Müller F. Virtual reality to augment robotassisted gait training in non-ambulatory patients with a subacute stroke: a pilot randomized controlled trial. European journal of physical and rehabilitation medicine June: 54(3), 397-407. (2018). https://doi.org/10.23736/S19739087.17.04735-9

10. Rooij IJMD, Port IGLVD, Meijer JWG. Effect of Virtual Reality Training on Balance and Gait Ability in Patients With Stroke: Systematic Review and Meta-Analysis. Physical therapy, 96(12), 1905-1918.

https://doi.org/10.2522/ptj.20160054

(2016).

11. In T, Lee K, Song C. Virtual Reality Reflection Therapy Improves Balance and Gait in Patients with Chronic Stroke: Randomized Controlled Trials. Medical Science Monitor, 40464053. (2016). https://doi:10.12659/MSM.898157

12. Kalron A, Fonkatz I, Frid L, Baransi H, Achiron A. The effect of balance training on postural control in people with multiple sclerosis using the CAREN virtual reality system: a pilot randomized controlled trial. Journal of NeuroEngineering and Rehabilitation, 13(1),13. (2016) https://doi:10.1186/s12984-016-0124-y

13. Kim A, Darakjian N, Finley JM. Walking in fully immersive virtual environments: an evaluation of potential adverse effects in older adults and individuals with Parkinson's disease. Journal of NeuroEngineering and Rehabilitation, 14(1),16. (2017) https://doi:10.1186/s12984-017-0225-2 https://doi:10.1155/2019/7595639

14. Severiano MIR, Zeigelboim BS, Teive HAG, Santos GJB, Fonseca VR. Effect of virtual reality in Parkinson's disease: a prospective observational study. Arquivos de Neuro-Psiquiatria. 76(2), 7884. (2018) https://dx.doi.org/10.1590/0004282x20170195

15. Yang WC, Wang HK, Wu RM, Lo CS, Lin KH. Home-based virtual reality balance training and conventional balance training in Parkinson's disease: A randomized controlled trial. Journal of the Formosan Medical Association. Sep;115(9):734-43, (2015) https://doi:10.1016/j.jfma.2015.07.012 